Europhys. Lett., 57 (2), pp. 171-177 (2002)

\title{
Memory effects in the relaxation of Ising models
}

\author{
J. J. Brey and A. Prados \\ Física Teórica, Universidad de Sevilla \\ Apartado de Correos 1065, E-41080, Sevilla, Spain
}

(received 14 May 2001; accepted in final form 2 November 2001)

PACS. 05.50.+q - Lattice theory and statistics (Ising, Potts, etc.).

PACS. 05.70.Ln - Nonequilibrium and irreversible thermodynamics.

PACS. 45.70.Cc - Static sandpiles; granular compaction.

\begin{abstract}
It is analytically shown that the one-dimensional Ising model with Glauber dynamics exhibits short-time memory effects when submitted to an abrupt change in temperature. These effects are qualitatively similar to those experimentally observed in the compaction of vibrated granular materials. Moreover, a critical time separating regimes of "normal" and "anomalous" responses to the perturbation is found.
\end{abstract}

The dynamics of granular systems exhibits a rich phenomenology that has been the subject of intensive study in the last years [1]. In particular, when submitted to a vertical vibration starting from a loose packing configuration, granular media compact behaving in a way that is reminiscent of conventional structural glasses $[2,3]$. The glassy nature of granular compaction clearly shows up when the response of the system to a sudden change in the vibration intensity is measured [4-6]. The results show the presence of relevant short-term memory effects that are in contrast with the long-time behaviour observed at constant intensity. A decrease (increase) in the vibration acceleration leads to an increase (decrease) of the compaction rate at short times. For large times, the previous history is forgotten and the relaxation rate becomes the same as for a constant-intensity process.

Here we study the existence of a similar "anomalous" response in the context of the one-dimensional Ising model with Glauber dynamics [7]. This is a model with short-range interactions that exhibits many of the qualitative features of conventional structural glasses, like slow non-exponential relaxation, a kinetic phenomenon resembling a laboratory glass transition, aging, and hysteresis effects [8-19]. On the other hand, it is simple enough to allow exact analytical calculations in situations where more realistic models are untractable. In particular, we will be able to calculate the short-time response of the system to a sudden small change in temperature, which plays in the model a role analogous to the vibration intensity in granular compaction. As long as the change is not made at the early stages of the relaxation process, the system shows an "anomalous" response. On the other hand, if the perturbation is introduced very soon, the response is "normal", in the sense that the modification of the relaxation rate has the same sign as the temperature change. Let us mention that these two qualitatively different time regimes have been also identified within a 
general theoretical framework developed to analyze memory effects in granular compaction [6], although it has not been experimentally verified yet to the best of our knowledge.

The energy of the one-dimensional Ising model in the configuration $\boldsymbol{\sigma} \equiv\left\{\sigma_{i}\right\}, \sigma_{i}= \pm 1$, is

$$
\mathcal{H}(\boldsymbol{\sigma})=-J \sum_{k}\left(\sigma_{k} \sigma_{k+1}-1\right)
$$

where $J>0$ is the ferromagnetic coupling constant between nearest-neighbour spins. The Glauber dynamics for the model is formulated by means of a master equation, with the transition rate from configuration $\boldsymbol{\sigma}$ to configuration $R_{k} \boldsymbol{\sigma}$, differing from $\boldsymbol{\sigma}$ only in the flip of spin $\sigma_{k}$, given by [7]

$$
\omega_{k}(\boldsymbol{\sigma})=\frac{\alpha}{2}\left[1-\frac{\gamma}{2} \sigma_{k}\left(\sigma_{k-1}+\sigma_{k+1}\right)\right] .
$$

Here

$$
\gamma=\tanh \frac{2 J}{k_{\mathrm{B}} T}
$$

$k_{\mathrm{B}}$ being Boltzmann's constant and $T$ the temperature of the system. The quantity $\alpha$ determines the global time scale of the system. Although it can be taken as temperature independent, in the context of structural glasses, where spin configurations are associated with the minima of the potential energy [14], an Arrhenius expression

$$
\alpha(T)=\alpha_{0} \exp \left[-\frac{B}{k_{\mathrm{B}} T}\right],
$$

is often considered $[15,20]$. The constant $B$ is a measure of the energy barrier separating energy minima in configuration space and $\alpha_{0}$ can be employed to define a dimensionless basic time scale. In the following, we will use eq. (4) for the sake of generality. The particular case of temperature-independent $\alpha$ can be obtained by taking the limit $B \rightarrow 0$.

We will restrict ourselves to homogeneous situations, i.e. states where all the averages are translationally invariant. It is useful to introduce the spin-spin correlations

$$
C_{n}(t) \equiv\left\langle\sigma_{k} \sigma_{k+n}\right\rangle_{t}=\sum_{\boldsymbol{\sigma}} \sigma_{k} \sigma_{k+n} p(\boldsymbol{\sigma}, t)
$$

where $p(\boldsymbol{\sigma}, t)$ is the probability of configuration $\boldsymbol{\sigma}$ at time $t$. In an infinite lattice, the time evolution of these correlations at constant temperature is given by [7]

$$
\frac{\mathrm{d}}{\mathrm{d} t} C_{n}=-2 \alpha C_{n}+\alpha \gamma\left(C_{n-1}+C_{n+1}\right)
$$

for $n \geq 1$, while $C_{0}=1$. The equilibrium correlations are

$$
C_{n}^{(s)}(T)=\xi(T)^{n}, \quad \xi(T)=\tanh \frac{J}{k_{\mathrm{B}} T} .
$$

Denoting by $N$ the number of spins, a dimensionless energy per spin can be defined as

$$
E(t) \equiv \frac{\langle\mathcal{H}\rangle_{t}}{N J}=1-C_{1}(t)
$$

An equation for the time evolution of $E(t)$ follows by particularizing eq. (6) for $n=1$. We will write it as an expression for the instantaneous energy relaxation rate $r(t)$,

$$
r(t) \equiv-\frac{\mathrm{d} E(t)}{\mathrm{d} t}=\alpha(T)\left[\mu_{1}(t)-\varepsilon(T) \mu_{2}(t)\right]
$$


where

$$
\mu_{1}(t)=2 E(t)-1+C_{2}(t) \geq 0, \quad \mu_{2}(t)=1+C_{2}(t) \geq 0,
$$

and we have introduced

$$
\varepsilon(T)=1-\gamma(T) .
$$

Both $\varepsilon(T)$ and $\alpha(T)$ are monotonic increasing functions of the temperature. An equation having the same structure as eq. (9) was proposed to describe compaction in tapped granular systems in ref. [6]. The main difference is that here we have considered the time evolution of the energy, instead of the density. In the interpretation of the Ising model as describing structural relaxation, an increase of the density corresponds to a decrease of the energy. This is the reason why we have defined $r(t)$ with a minus sign in eq. (9).

Let us suppose we carry out the following experiment. Starting from a given configuration, the system is allowed to relax at constant temperature $T$. At a certain time $t_{w}$, before the system has reached the steady configuration corresponding to $T$, the temperature is suddenly changed to $T+\Delta T$. This produces a jump $\Delta r_{w}$ in the relaxation rate. For $\Delta T \ll T$, keeping only first-order terms in the perturbation $\Delta T$, it is easily obtained that

$$
\Delta r_{w}=\lambda\left(t_{w}\right) \Delta \alpha
$$

with

$$
\lambda(t)=\frac{r(t)}{\alpha}-\alpha \mu_{2}(t) \frac{\mathrm{d} \varepsilon}{\mathrm{d} \alpha}=\frac{r(t)}{\alpha}-\frac{\eta\left(1-\gamma^{2}\right)}{2} \mu_{2}(t),
$$

and $\eta=4 J / B$. The function $\lambda(t)$ is defined along the relaxation curve at constant $T$. As long as $\Delta T$ is small enough so that the linear approximation we are considering is accurate, the function $\lambda(t)$ for $t=t_{w}$ determines the relative behaviour of the relaxation rate jump $\Delta r_{w}$ with respect to $\Delta \alpha$ (or $\Delta T)$.

To study the function $\lambda(t)$, we have to evaluate the relaxation rate $r(t)$ and the correlation function $\mu_{2}(t)$ for a process at constant temperature. The general solution of the hierarchy (6) for an arbitrary initial condition can be analytically found [7,21]. We will consider processes starting in the state of maximum disorder, i.e. the equilibrium state at infinite temperature. Similar results are obtained for other initial conditions, as long as they correspond to highly disordered states. A simple calculation gives [7,21]

$$
\Delta_{n}(t) \equiv C_{n}(t)-C_{n}^{(s)}(T)=-\frac{\gamma}{\pi} \int_{0}^{\pi} \mathrm{d} q \frac{\sin q \sin n q}{1-\gamma \cos q} e^{-2 \alpha t(1-\gamma \cos q)} .
$$

Use of the above expression with $n=1$ into eq. (8) yields

$$
E(t)-E^{(s)}(T)=\frac{\gamma}{\pi} \int_{0}^{\pi} \mathrm{d} q \frac{\sin ^{2} q}{1-\gamma \cos q} e^{-2 \alpha t(1-\gamma \cos q)},
$$

where $E^{(s)}(T)=1-C_{1}^{(s)}(T)$ is the equilibrium value of the dimensionless energy per particle. The relaxation rate of the energy, defined in eq. (9), for the present process is

$$
r(t)=\frac{2 \alpha \gamma}{\pi} \int_{0}^{\pi} \mathrm{d} q \sin ^{2} q e^{-2 \alpha t(1-\gamma \cos q)}=\frac{e^{-2 \alpha t} I_{1}(2 \alpha \gamma t)}{t},
$$

where $I_{1}$ is the modified Bessel function of the first kind [22].

To evaluate $\mu_{2}(t)$ we use again eq. (14), now for $n=2$. In this way we get from eq. (10)

$$
\mu_{2}(t)=1+\xi^{2}-\frac{\gamma}{\pi} f(t)
$$


where

$$
f(t)=\int_{0}^{\pi} \mathrm{d} q \frac{\sin q \sin 2 q}{1-\gamma \cos q} e^{-2 \alpha t(1-\gamma \cos q)} .
$$

Substitution of eqs. (16) and (17) into eq. (13) leads to

$$
\lambda(t)=\frac{e^{-2 \alpha t} I_{1}(2 \alpha \gamma t)}{\alpha t}-\frac{\eta\left(1-\gamma^{2}\right)}{2}\left[1+\xi^{2}-\frac{\gamma}{\pi} f(t)\right] .
$$

In the following we will consider that the temperature $T$ is very low, so that $\varepsilon \ll 1, \gamma \rightarrow 1$, $\xi \rightarrow 1$, and $\alpha \rightarrow 0$. It could be concluded that the second term in the right-hand side of eq. (19) can be neglected as compared with the first one [23] in this limit. Nevertheless, this is not true in general, and the relative relevance of both terms depends on the time scale of interest. In particular, for $t \rightarrow \infty$ the first term vanishes, as well as $f(t)$, and

$$
\lambda_{\infty} \equiv \lim _{t \rightarrow \infty} \lambda(t)=-\frac{\eta\left(1-\gamma^{2}\right)\left(1+\xi^{2}\right)}{2} \sim-2 \eta \varepsilon<0 .
$$

Since $\lambda(t)$ is a continuous function of $t$, the above result implies that, for large enough $t_{w}$, $\lambda\left(t_{w}\right)<0$ and, therefore, $\Delta r_{w}$ and $\Delta \alpha$ have opposite signs. A sudden increase of the temperature produces a negative jump of the relaxation rate. This is the kind of "anomalous" response found in compaction processes of granular materials [4-6].

It is convenient to define the function

$$
\Lambda(t)=\lambda(t)-\lambda_{\infty}=\frac{e^{-2 \alpha t} I_{1}(2 \alpha \gamma t)}{\alpha t}+\frac{\eta \gamma\left(1-\gamma^{2}\right)}{2 \pi} f(t),
$$

that vanishes for $t \rightarrow \infty$. We are going to analyze the behaviour of this quantity both in the short- and the large-time regions. First, consider "short" times characterized by

$$
\alpha t \sim O(1),
$$

so that $\varepsilon \alpha t \ll 1$ for the low temperatures we are dealing with. On the time scale defined above, we can approximate $\gamma$ by unity in the expression of $f(t)$, eq. (18), obtaining

$$
f(t)=\pi e^{-2 \alpha t}\left[I_{0}(2 \alpha t)+2 I_{1}(2 \alpha t)+I_{2}(2 \alpha t)\right] .
$$

When this expression is substituted into eq. (21), it is easily realized that the term involving $f(t)$ can be neglected because of the $\eta \varepsilon$ factor. Then,

$$
\Lambda(t) \sim \frac{e^{-2 \alpha t} I_{1}(2 \alpha t)}{\alpha t},
$$

for $\alpha t \sim O(1), \varepsilon \ll 1$. In this time window, $\lambda(t) \sim \Lambda(t)>0$, where we have taken into account that $\lambda_{\infty}$ is of order $\varepsilon$ (see eq. (20)). As a consequence, the response of the system to a small change $\Delta T$ in the temperature at $t=t_{w}$ is "normal" if $\alpha \varepsilon t_{w} \ll 1$. The modification of the relaxation rate has the same sign as the temperature change. Above, it was shown that for asymptotically large times the response was qualitatively different. Let us study in more detail what happens in the "large" time region. We define a slow time scale by

$$
\tau \equiv \alpha \varepsilon t \sim O(1),
$$

i.e., times of the order of the system relaxation time. For these times, it is $\alpha t=O\left(\varepsilon^{-1}\right) \gg 1$ and we can approximate $I_{1}(2 \alpha \gamma t)$ by its asymptotic behaviour for large argument [22], getting

$$
\frac{e^{-2 \alpha t} I_{1}(2 \alpha \gamma t)}{\alpha t} \sim \varepsilon^{3 / 2} \frac{e^{-2 \tau}}{2 \pi^{1 / 2} \tau^{3 / 2}} .
$$


This quantity is much smaller than $\lambda_{\infty}$. Moreover, a simple asymptotic analysis gives

$$
f(t) \sim(2 \pi \varepsilon)^{1 / 2} \Gamma(-1 / 2,2 \tau)
$$

$\Gamma(a, x)$ being the incomplete Gamma-function [22]. Use of eqs. (26) and (27) into eq. (21) leads to

$$
\Lambda(t) \sim \varepsilon^{3 / 2}\left[\frac{e^{-2 \tau}}{2 \pi^{1 / 2} \tau^{3 / 2}}+\eta\left(\frac{2}{\pi}\right)^{1 / 2} \Gamma(-1 / 2,2 \tau)\right],
$$

valid for $\varepsilon \ll 1, \tau=O(1)$. In this long-time regime, $\Lambda(t)$ is of the order of $\varepsilon^{3 / 2}$, and $\left|\lambda_{\infty}\right| \gg \Lambda(t)$. Then, $\lambda(t) \sim \lambda_{\infty}<0$. The response of the system is anomalous, as we already knew from the discussion of the asymptotic behaviour for $t \rightarrow \infty$.

Since we have found that $\lambda(t)$ has different signs in the short- and long-time regions, because of continuity reasons there must be at least one critical time $t_{\mathrm{c}}$ at which $\lambda\left(t_{\mathrm{c}}\right)=0$. This time separates regions with normal and anomalous behaviour. Furthermore, the above discussion indicates that this time must belong to an intermediate time scale between those previously considered, namely those given by eqs. (22) and (25), respectively. The matching time range where the two solutions, eqs. (24) and (28), are valid is characterized by

$$
\alpha t \gg 1, \quad \tau=\varepsilon \alpha t \ll 1 .
$$

On this time scale, we can substitute the Bessel function in eq. (24) by its asymptotic behaviour for large argument [22], with the result

$$
\Lambda(t) \sim \frac{1}{2 \pi^{1 / 2}(\alpha t)^{3 / 2}}
$$

The same expression is obtained from eq. (28) in the appropriate limit, showing that the solutions corresponding to the short- and large-time scales match in the intermediate scale defined by eq. (29). A single uniform approximation $\Lambda_{\text {unif }}(t)$, valid for all times, is obtained by adding eqs. (24) and (28), and subtracting the expression in the matching region, eq. (30),

$$
\Lambda_{\text {unif }}(t)=\frac{e^{-2 \alpha t} I_{1}(2 \alpha t)}{\alpha t}+\frac{e^{-2 \alpha \varepsilon t}-1}{2 \pi^{1 / 2}(\alpha t)^{3 / 2}}+\varepsilon^{3 / 2} \eta\left(\frac{2}{\pi}\right)^{1 / 2} \Gamma(-1 / 2,2 \alpha \varepsilon t) .
$$

In fig. 1 we compare this result with the exact expression, eq. (21) for $\varepsilon=0.01$ and $J=B$, i.e. $\eta=4$. A very good agreement is observed for all times. The relevant physical quantity we are interested in is the critical time $t_{\mathrm{c}}$, verifying $\lambda\left(t_{\mathrm{c}}\right)=0$ or, equivalently, $\Lambda\left(t_{\mathrm{c}}\right)=-\lambda_{\infty}$. This latter quantity is also indicated in the figure. Since $t_{c}$ is expected to belong to the intermediate scale, we get from eqs. (20) and (30)

$$
\alpha t_{\mathrm{c}} \sim\left(4 \pi^{1 / 2} \eta \varepsilon\right)^{-2 / 3} .
$$

This result confirms that $t_{\mathrm{c}}$ is in the overlapping region in agreement with our previous ansatz, since in the limit $\varepsilon \rightarrow 0$ it is $\alpha t_{\mathrm{c}} \gg 1$ and $\tau_{\mathrm{c}}=\varepsilon \alpha t_{\mathrm{c}} \ll 1$. Therefore, eq. (32) is consistent with the uniform expression (31). It is interesting to analyze the temperature dependence of $t_{\mathrm{c}}$. By taking logarithms in eq. (32), we find, for low $T$,

$$
\ln \left(\alpha_{0} t_{\mathrm{c}}\right)=-\frac{2}{3} \ln \left(8 \pi^{1 / 2} \eta\right)+\frac{B}{k_{\mathrm{B}} T}\left(1+\frac{2 \eta}{3}\right),
$$




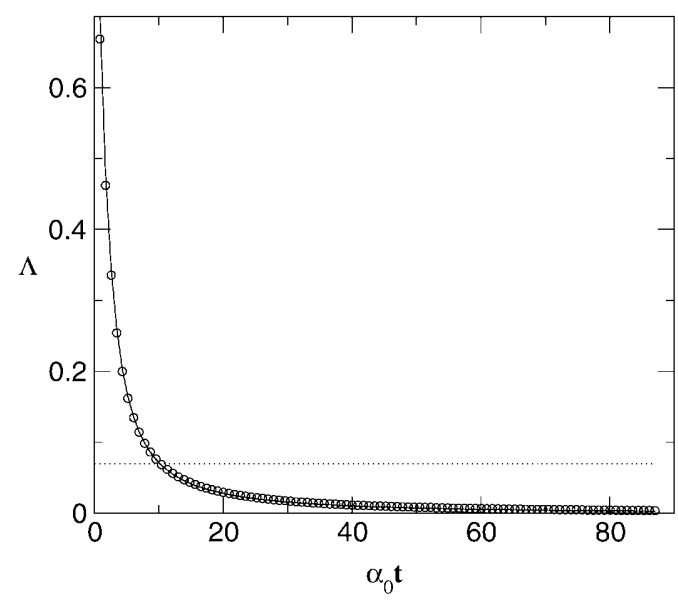

Fig. 1

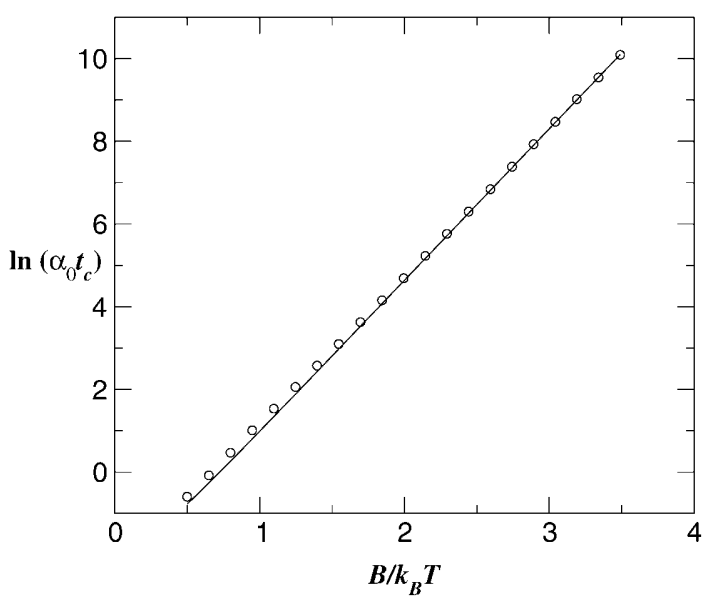

Fig. 2

Fig. 1 - Comparison of the numerical evaluation of eq. (21) (circles) with the uniform asymptotic approximation given by eq. (31) (solid line). Also plotted (dotted line) is the asymptotic long-time value $\left|\lambda_{\infty}\right|$. Note that the quantities represented in both axes are dimensionless.

Fig. 2 - The dimensionless critical time $t_{\mathrm{c}}$, separating the regions of normal and anomalous response, as a function of the inverse of the reduced temperature. The circles have been obtained by numerical evaluation of eq. (21), while the solid line is the result of the asymptotic calculation, eq. (32).

showing an Arrhenius dependence. In fig. 2 the logarithm of the critical time $t_{\mathrm{c}}$, given by eq. (32), is plotted as a function of the inverse of the reduced temperature $B / k_{\mathrm{B}} T$, again for $\eta=4$. For comparison, also the numerical values obtained from the exact expression, eq. (21), are included. A linear profile, consistent with eq. (33), is observed.

Let us now address the particular case of $\alpha$ being temperature independent that can be obtained by considering the limit $B \rightarrow 0$. Then $\eta$ goes to infinity and $t_{\mathrm{c}}$ vanishes. Therefore, the system always exhibits an anomalous response to a temperature jump, independently of the time instant in which it is produced. The physical reason for this result is clear, since eq. (12) reduces in this situation to

$$
\frac{\Delta r_{w}}{\Delta T}=-\alpha \mu_{2 w} \frac{\mathrm{d} \varepsilon}{\mathrm{d} T}<0 .
$$

Equation (34) reflects that, if $\alpha$ does not depend on the temperature, the rates of the processes decreasing the energy do not change instantaneously in a temperature jump. This can be directly verified, for instance, from eq. (9).

In conclusion, the major result obtained in this paper is the analytical derivation of memory effects in the relaxation of the one-dimensional Ising model with Glauber dynamics. Moreover, these effects are qualitatively similar to those observed experimentally in granular compaction [4-6], for which the existence of a critical time separating the regions of "normal" and "anomalous" responses has been theoretically argued [6]. Therefore, also in the present context the Ising model seems to be the simplest relevant system beyond mean-field descriptions. The results in this paper seem to indicate that the memory effects and the presence of a critical time, as discussed here, may be quite general. In the Ising model, the critical time lies in an intermediate time window, being much smaller than the average relaxation time of the system towards its equilibrium state. 
We acknowledge partial support from the Dirección General de Investigación Científica y Técnica (Spain) through Grant No. PB98-1124.

\section{REFERENCES}

[1] Jaeger H., Nagel S. R. and Behringer R., Rev. Mod. Phys., 68 (1996) 1259.

[2] Knight J. B., Frandich C. G., Lau C. N., Jaeger H. M. and Nagel S. R., Phys. Rev. E, 51 (1995) 3957.

[3] Nowak E. R., Knight J. B., Ben-Naim E., Jaeger H. M. and Nagel S. R., Phys. Rev. E, 57 (1998) 1971.

[4] Nicodemi M., Phys. Rev. Lett., 82 (1999) 3734.

[5] Josserand C., Tkachenko A., Mueth D. M. and Jaeger H. M., Phys. Rev. Lett., 85 (2000) 3632 .

[6] Brey J. J. and Prados A., Phys. Rev. E, 63 (2001) 061301.

[7] Glauber R. J., J. Math. Phys., 4 (1963) 294.

[8] Anderson J. E., J. Chem. Phys., 52 (1970) 2821.

[9] Skinner J. L., J. Chem. Phys., 79 (1983) 1955.

[10] Budimir J. and Skinner J. L., J. Chem. Phys., 82 (1985) 5232.

[11] Spohn H., Commun. Math. Phys., 125 (1989) 3.

[12] Brey J. J. and Prados A., Physica A, 197 (1993) 569.

[13] Brey J. J. and Prados A., Phys. Rev. E, 53 (1996) 458.

[14] Kob W. and Schilling R., J. Phys. A, 23 (1990) 4673; Phys. Rev. A, 42 (1990) 2191.

[15] Brey J. J. and Prados A., Phys. Rev. B, 49 (1994) 984.

[16] Bray A. J., J. Phys. A, 23 (1990) L67.

[17] Prados A., Brey J. J. and Sánchez-Rey B., Europhys. Lett., 40 (1997) 13.

[18] Lippiello E. and Zannetti M., Phys. Rev. E, 61 (2000) 3369.

[19] Godrèche C. and Luck J. M., J. Phys. A, 33 (2000) 1151.

[20] Schilling R., J. Stat. Phys., 53 (1988) 1227.

[21] Bedeaux D., Shuler K. E. and Oppenheim I., J. Stat. Phys., 2 (1970) 1.

[22] Abramowitz M. and Stegun I. A. (Editors), Handbook of Mathematical Functions (Dover, New York) 1965.

[23] Note that $I_{1}(z) / z \rightarrow 1 / 2$ for $z \rightarrow 0[22]$. 